\title{
Commentary on: Validation of sentinel lymph node dissection in prostate cancer: experience in more than 2,000 patients
}

\author{
Isabelle Brenot-Rossi • Cyrille Bastide
}

Published online: 23 June 2009

(C) Springer-Verlag 2009

Lymph node metastases are one of the most important prognostic factors in prostate cancer. $\mathrm{N}$ staging directly influences the treatment decision in patients for whom potentially curative treatments are planned. However, the authors [1] provide a reminder that there is an ongoing debate about which patients require lymph node resection and how the dissection should be extended [2]. The Augsburg group first developed and described sentinel lymphadenectomy (SLNE) in prostate cancer. They have demonstrated through some studies that the majority of sentinel lymph node (SLN) and non-SLN metastases were found along the internal iliac vessels and that limited lymphadenectomy to obturator fossa was clearly insufficient for accurate lymph node staging. These findings are sustained by the majority of studies reporting on extended pelvic lymphadenectomy in prostate cancer. In this study, they reported the largest series with more than 2,000 patients who underwent SLNE alone or in combination with either standard or extended lymphadenectomy. It is the most important published manuscript in the literature. However, this study gathers heterogeneous subgroups of patients. The first goal of the study was to evaluate the

This Editorial Commentary refers to the article http://dx.doi.org/ 10.1007/s00259-009-1157-2

\footnotetext{
I. Brenot-Rossi $(\bowtie)$

Department of Nuclear Medicine, Regional Cancer Center,

Institut Paoli-Calmettes,

Marseilles, France

e-mail: brenotrossii@marseille.fnclcc.fr

C. Bastide

Department of Urology, Université de la Méditerranée,

CHU Nord,

Marseille, France
}

false-negative rate (number of false-negative SLN/total number of positive nodes) of the sentinel node procedure, but only $30 \%$ of the patients underwent sentinel lymph node resection associated with either standard or extended lymphadenectomy. So, the false-negative rate could be evaluated from only 606 patients: those with standard lymphadenectomy (lymph nodes removed along the external iliac vessels and the obturator nerve) and those with extended lymphadenectomy (including also lymph nodes of the internal iliac vessels and their branches). In those conditions, this result is critical, but remains interesting because it was obtained from a high number of men. As already demonstrated, probably many factors such as sufficient injected activity are necessary to avoid failure of the SLN procedure and so get a low false-negative rate [3]. In this study, the Augsburg team found a false-negative rate of less than $6 \%$, which is within the limits set by international guidelines for breast carcinoma.

More interesting, the authors observed that the falsenegative rate grows in relation to pathological Gleason score. They defined several subgroups of patients according to Gleason score of the prostate biopsy, in which the sentinel lymph node procedure could be realised. It is one of the most important conclusions given by these authors; however, some comments and remarks may be suggested. After the validation period including 400 patients, the authors changed the lymphadenectomy modalities and proposed SLNE only for patients with a biopsy Gleason score up to $3+4$, because the risk of lymphatic metastasis spread is low. On the other hand, patients belonging to the subgroup of patients with biopsy Gleason score $7(4+3)$ and score 8 underwent SLNE associated with an extended lymphadenectomy. In this case, the surgical procedure is a radioguided surgery instead of the SLN procedure [4]. Moreover, patients with a Gleason score higher than 
8 underwent extended lymphadenectomy only, because they have a high risk of lymph node metastases, associated with a high false-negative rate of $14 \%$; in this case, the benefit of the SLN procedure remains an open question in patients with very high risks of metastases.

This proposition of risk groups for lymph nodes metastases, according to biopsy Gleason score, appears insufficient and questionable. Indeed, the pre-treatment level of serum prostate-specific antigen (PSA) is a very important risk factor for lymph node metastases. It seems difficult to evaluate the risk of lymph node metastasis only with the biopsy Gleason score. Furthermore, the risk of undergrading between biopsy Gleason score and specimen Gleason score is not negligible [5].

Regarding the false-negative rate of $14 \%(6 / 43)$ in patients with Gleason score $>8$, it is important to underline that all of six patients had lymph node metastasis $>1 \mathrm{~cm}$ probably detected by CT scan. This point is very interesting and the authors are right to write that patients with large lymph nodes visible on $\mathrm{CT}$ scan are probably bad candidates for SLNE. It would be interesting to know the mean number of lymph nodes removed per patient according to lymphadenectomy performed.

The authors reported a SLNE sensitivity of $94 \%$ for detection of lymph node metastasis in 187 patients. These results are very encouraging and should help to redefine the definition of standard pelvic lymphadenectomy in prostate cancer which differs according to different authors. Indeed, as used by the MSKCC group (Memorial Sloan-Kettering Cancer Center, New York [6]) templates of limited pelvic lymph node dissection include the external iliac lymph nodes, whereas standard pelvic lymph node dissection includes the external iliac, obturator and internal iliac lymph nodes. This point is very important to understand the results of different series.

In conclusion, the authors proposed new updated nomograms about the need for SLN resection with or without non-SLN pelvic dissection according to the Gleason score. Regarding the place for SLNE in pelvic node staging, it seems very interesting to associate SLNE with standard lymphadenectomy for patients with localised prostate cancer and risk factors for lymph node metastases (Gleason $\geq 7$ and pre-treatment PSA $>10 \mathrm{ng} / \mathrm{ml}$ ). SLNE only should be reserved for low-risk patients.

\section{References}

1. Holl G, Dorn R, Wengenmair H, Weckermann D, Sciuk J. Validation of sentinel lymph node dissection in prostate cancer: experience in more than 2,000 patients. Eur J Nucl Med Mol Imaging 2009. doi: 10.1007/s00259-009-1157-2.

2. Heidenreich A (chairman), Bolla M, Joniau S, van der Kwast TH, Matveev V, Mason MD et al. European Association of Urology 2009. Guidelines on prostate cancer 2009.

3. Brenot-Rossi I, Rossi D, Esterni B, Brunelle S, Chuto G, Bastide C. Radioguided sentinel lymph node dissection in patients with localised prostate carcinoma: influence of the dose of radiolabelled colloid to avoid failure of the procedure. Eur J Nucl Med Mol Imaging 2008;35:32-8.

4. Bastide C, Brenot-Rossi I, Garcia S, Rossi D. Radioisotope guided sentinel lymph node dissection in patients with localized prostate cancer: results of the first 100 cases. Eur J Surg Oncol 2009;35:751-6.

5. Cookson MS, Fleshner NE, Soloway SM, Fair WR. Correlation between Gleason score of needle biopsy and radical prostatectomy specimen: accuracy and clinical implications. J Urol 1997;157:559-62.

6. Touijer K, Rabbani F, Otero JR, Secin FP, Eastham JA, Scardino PT, et al. Standard versus limited pelvic lymph node dissection for prostate cancer in patients with a predicted probability of nodal metastasis greater than 1\%. J Urol 2007;178:120-4. 